\title{
A NOVEL APPROACH OF CLUSTERING FOR ENHANCED LIFETIME IN WIRELESS SENSOR NETWORK
}

\author{
Chamanpreet Kaur (1), Vikramjit Singh (2) \\ (1) Research Scholar, Department of Computer Science and Engineering, NWIET, Moga \\ (2) Assistant Professor, Department of Computer Science and Engineering, NWIET, Moga
}

\begin{abstract}
Wireless sensor network has revolutionized the way computing and software services are delivered to the clients on demand. Our research work proposed a new method for cluster head selection having less computational complexity. It was also found that the modified approach has improved performance to that of the other clustering approaches. The cluster head election mechanism will include various parameters like maximum residual energy of a node, minimum separation distance and minimum distance to the mobile node. Each $\mathrm{CH}$ will create a TDMA schedule for the member nodes to transmit the data. Nodes will have various level of power for signal amplification. The three levels of power are used for amplifying the signal. As the member node will send only its own data to the cluster head, the power level of the member node is set to low. The cluster head will send the data of the whole cluster to the mobile node, therefore the power level of the cluster head is set to medium. High power level is used for mobile node which will send the data of the complete sector to the base station. Using low energy level for intra cluster transmissions (within the cluster) with respect to cluster head to mobile node transmission leads in saving much amount of energy. Moreover, multi-power levels also reduce the packet drop ratio, collisions and/ or interference for other signals. It was found that the proposed algorithm gives a much improved network lifetime as compared to existing work. Based on our model, multiple experiments have been conducted using different values of initial energy.
\end{abstract}

\section{Keywords}

Wireless Sensor Network, Energy saving, Protocol, Cluster Head, Node, WSN.

\section{INTRODUCTION}

A Wireless Sensor Network or WSN is supposed to be made up of a large number of sensors and at least one base station. The sensors are autonomous small devices with several constraints like the battery power, computation capacity, communication range and memory. They also are supplied with transceivers to gather information from its environment and pass it on up to a certain base station, where the measured parameters can be stored and available for the end user. In most cases, the sensors forming these networks are deployed randomly and left unattended to and are expected to perform their mission properly and efficiently. As a result of this random deployment, the WSN has usually varying degrees of node density along its area. Sensor networks are also energy constrained since the individual sensors, which the network is formed with, are extremely energy-constrained as well. The communication devices on these sensors are small and have limited power and range. Both the probably difference of node density among some regions of the network and the energy constraint of the sensor nodes cause nodes slowly die making the network less dense. Also, it is quite common to deploy WSNs in harsh environment, what makes many sensors inoperable or faulty. For that reason, these networks need to be fault-tolerant so that the need for maintenance is minimized. Typically, the network topology is continuously and dynamically changing, and it is actually not a desired solution to replenish it by infusing new sensors instead the depleted ones. A real and appropriate solution for this problem is to implement routing protocols that perform efficiently and utilizing the less amount of energy as possible for the communication among nodes. Due to recent technological advances, the manufacturing of small and low cost sensors became technically and economically feasible. The sensing electronics measure ambient conditions related to the environment surrounding the sensor and transform them into an electric signal. Processing such a signal reveals some properties about objects located and/or events happening in the vicinity of the sensor. A large number of these disposable sensors can be networked in many applications that require unattended operations. A Wireless Sensor Network (WSN) contains hundreds or thousands of these sensor nodes. These sensors have the ability to communicate either among each other or directly to an external base-station (BS). Wireless Sensor Network (WSN) is a type of wireless Ad-Hoc network in which large numbers of sensor nodes are deployed in the application field [1].

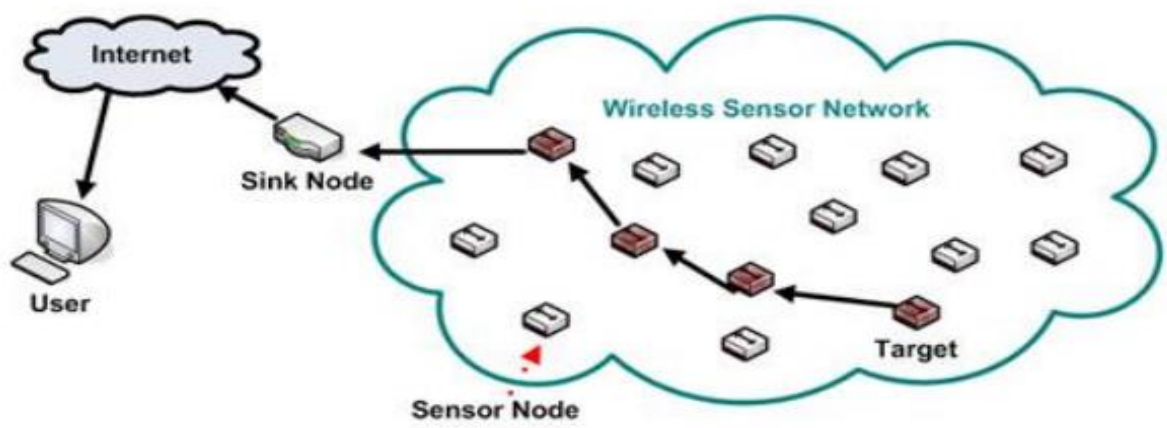

Figure 1. WSN Components 


\section{RELATED WORK}

The needed detailed literature survey, to get preliminary knowledge and search scope of investigation, to implement clustering is explained in this section. This section presents investigational studies in several energy efficient routing algorithms and its general purpose. Yu Xiuwu et al (2016) optimizes the energy-balanced problem and lengthen the network lifetime for Wireless sensor network (WSN) monitoring, a routing protocol that based on monitoring area partition clustering for energy-balanced (EBPC) was proposed. They divided monitoring area into several virtual partitions, controlled the number of common nodes and clusters by data fusion rate, and forwarded cluster heads data to the next partitions with minimum path selection coefficient. Dan Liu et al (2016) reduces the energy consumption and balance the traffic load throughout the wireless sensor networks (WSN). Due to the fault tolerance and traffic load balancing of the clustering method, CS is always combined with clustering for further improvement. And hexagon clustering has some advantages over other clustering methods such as its special structure. Zhu Yafei et al (2016) ensures the integrity and privacy of data transmission in wireless sensor network, by analyzing the existing security communication schemes. Samaresh Bera et al (2016) proposes a software-defined wireless sensor network architecture (Soft-WSN)—an effort to support applicationaware service provisioning in Internet of Things (IOT). Detailed architecture of the proposed system is presented involving the application, control, and infrastructure layers to enable software-defined networking (SDN) in loT. We design a software defined controller, which includes two management policies device management and network management. Device management facilitates users to control their devices in the network. To enable device control mechanisms, we investigate three scheduling issues in a sensor node-sensing task, sensing delay, and active sleep. Alduais et al (2016) states WSN possess several constraints such as limited energy availability, low memory size, and low processing speed, which are the principal obstacles to designing efficient management protocols for WSNs, what more if it concerns WSN-IOT integration. In IOT based WSN, the basic issues are concerned with the mechanism to reduce the energy consumption of nodes which will result in prolonging the lifetime of the nodes. It is a very significant consideration since the energy consumed in transmitting one bit via WSN is higher than running numerous microcontroller instructions. Data reduction approaches are used to achieve the least energy consumption and thus prolonging the lifetime of WSN.Omkar Singh et al (2016) considers the homogeneous network scenario where each node has same capability in terms of processing power, storage, energy etc. In the real scenario, nodes have different values of processing time, storage and energy. Therefore, it is essential to judge the performance of the well accepted routing protocols for heterogeneous wireless sensor networks (H-WSN). Nodes in WSN are dispersed in two ways viz advanced nodes (high energy) and normal nodes. Rani Kumari et al (2016) analyzes the performance of the various routing protocols of the wireless network in the WBAN and WSN. Moreover, the paper also compares their performance in the same network and in different network using different parameters i.e. PDR, latency and throughput etc. The paper signifies that the wireless protocols needs updating to perform well in the WBAN. Hector Kaschel et al (2016) states that the Wireless Sensor Network (WSN), have been technological advances that have improved some features to achieve low cost, low power consumption and tiny sizes in wireless sensor nodes. However, one of the issues still restless in Wireless Sensor Networks is the limited battery power of sensor nodes. Asdianur et al (2016) studied effectiveness in using Compressive Sensing (CS) algorithm in order to reduce measuring in IEEE 802.15.4 Standard Wireless Sensor Network (WSN). As well known, in common WSN work system, Base Station (BS) gather some information from available nodes, which the process itself consumes a lot of energy from each node. Mohd Zaki Shahabuddin et al (2016) proposes fundamental modelling of topology control algorithm to conserve individual WSN node's energy, and at the same time preserving the graph connectivity. The proposed topology control algorithm consists of three phases: 1 . Identifying connecting nodes at maximum transmission, 2.Pairing nodes with shortest algorithm/minimum energy level, 3 . Calculating/setting minimum power transmission per-node for energy conservation. The algorithm works-out locally and dispenses full graph connectivity, and theoretically would be able to reduce WSN control overhead. M. Shaiful Azrin et al (2016) implements a HTTP string at sensor nodes to allow devices in the constrained network to push its data to the targeted server, with the help of gateway to make the request. This paper used ZigBee connectivity in wireless sensor network environment to transfer sensor reading, collected by the sensor node to the smartphone that act as a gateway to make the HTTP request to the targeted server, which is for this paper is to be stored in Google Fusion Tables (GFT) server. The developed system was tested at laboratory and results are encouraging.

\section{RESEARCH GAP}

The sensor nodes are battery-powered devices, hence have limited energy. A large amount of energy is consumed during data transmission. Furthermore, a significant amount of energy is consumed during the route discovery and its maintenance phase. The lifetime of the network directly depends on the total energy consumption by each node. If a sensor node's energy reaches below a certain level, it will become nonfunctional and affects the performance of the network. Therefore, it is a big challenge for a routing protocol designer to manage the energy of the sensor nodes to maximize the network lifetime. Generally, WSN consists of a large number of sensor nodes, which makes the bandwidth allocation for each link very challenging. Moreover, in the process of route discovery and maintenance, an enormous amount of control packets has to be broadcasted among the sensor nodes. Thus, the network bandwidth allocation process depends on the number of links and the amount of data they can communicate. Sensor nodes are tiny embedded devices having limited processing and storage capacity. Therefore, the researchers have to design a light-weight routing protocol that does not have complicated computing procedures and functions. Hence, the sensor nodes can process and store the data efficiently.

- To decide cluster area on the basis of chosen cluster head consumes huge amount of energy resources in the communication between nodes for the purpose of fixing the cluster region and its head. When two cluster heads are very close to each other that means their ranges will overlap each other which will create a major problem.

- $\quad$ No parameter has been explained for the election of Cluster head. There are several parameters that are involved in the election of cluster head. 
- Same level of power is used to amplify the signals from member node to cluster head, cluster head to mobile node and mobile node to base station. This will consume lot of energy. Mobile node requires large amount of energy for transferring the data of complete sector to the base station.

\section{RESEARCH OBJECTIVES}

WSNs must offer characteristics such as reliability, availability and maintainability. Availability to a large extend depends on fault tolerance to keep the system working as expected.

- To review the existing routing algorithms in wireless sensor networks.

- To propose an enhanced energy efficient algorithm with maximum life span and throughput.

- To introduce the various levels of power for signal amplification.

- To elect the cluster head based on various parameters like maximum residual energy, minimum separation distance and minimum distance to mobile node.

- $\quad$ To simulate the proposed algorithm in Matlab environment and evaluate the results with the existing work.

\section{RESEARCH METHODLOGY}

- Sensor nodes are randomly deployed in the network. Different network areas have been used for experimental purposes. The base station is located outside the network.

- $\quad$ All nodes sends its energy level and location information to the sink, which divides network into clusters and further divided into sectors based on number of cluster groups. Each sector is assigned a mobile node.

- $\quad$ The $\mathrm{CH}$ is selected by using hybrid model of both centralized and distributed election mechanism. The cluster head election mechanism will include various parameters like maximum residual energy of a node, minimum separation distance and minimum distance to the mobile node. Each $\mathrm{CH}$ will create a TDMA schedule for the member nodes to transmit the data.

- $\quad$ Nodes will have various level of power for signal amplification. The three levels of power are used for amplifying the signal. As the member node will send only its own data to the cluster head, the power level of the member node is set to low. The cluster head will send the data of the whole cluster to the mobile node, therefore the power level of the cluster head is set to medium. High power level is used for mobile node which will send the data of the complete sector to the base station. Using low energy level for intra cluster transmissions (within the cluster) with respect to cluster head to mobile node transmission leads in saving much amount of energy. Moreover, multi-power levels also reduce the packet drop ratio, collisions and/ or interference for other signals.

The proposed mechanism operations can be divided into two phases:-

\section{Setup phase}

\section{Steady phase}

In the setup phase, the clusters are formed and a cluster-head is chosen for each cluster or the region inside the sector While in the steady phase, data is sensed and sent to the central base station. The steady phase is longer than the setup phase. This is done in order to minimize the overhead cost.

1. CH Selection: - During the setup phase, a predetermined fraction of nodes, $p$, choose themselves as cluster-heads. This is done according to a threshold value, $T(n)$. The threshold value depends upon the desired percentage to become a clusterhead- $p$, the current round $r$, and the set of nodes that have not become the cluster-head in the last $1 / p$ rounds, which is denoted by $G$. The formulae is as follows: $T(n)=p / 1-p[r \bmod (1 / p)]$ if $n E G T(n)=0$ otherwise every node wanting to be the cluster-head chooses a value, between 0 and 1 . If this random number is less than the threshold value, $T(n)$, then the node becomes the cluster-head for the current round

2) Sleep mechanism implementation: - In WSN, some nodes in a cluster may far from its cluster head compared to other nodes in the cluster due to geographic location, terrain or distribution of nodes. Since these nodes deplete more energy than other nodes in the cluster while communicating with the head nodes, they will die much earlier and lead to the partition of the network. According to the proposed scheme, once the nodes have elected themselves to be $\mathrm{CHs}$ using the probabilities above, $\mathrm{CH}$ node broadcasts an advertisement message ( $\mathrm{msg}$ ). This message contains the node's identity (ID) and a header that distinguishes this message as an announcement message. Each non-cluster head node determines its cluster for this round by choosing the cluster head that requires the minimum communication energy, based on the received signal strength of the advertisement from each cluster head and then transmits a join-request message back to the chosen cluster head. This message consists of the current energy of the node, cluster message head's ID and the node's ID. Cluster head will check the picked nodes according to the order of the ranking, and then $\mathrm{n}$ will be added to the sleep nodes list and the ID of residual energy member node, for a node $n$, if this node will be deleted in all the $\mathrm{D}$ of other nodes. The $\mathrm{CH}$ node will broadcast a command message mgs to its cluster members while the checking complete. This message contains the sleep nodes list in which includes the ID of nodes that are ordered to sleep. These nodes will switch into sleep-mode, cease their communication with cluster heads and only sense the network status. The non-sleep nodes in the cluster return an acknowledgement message to the head node. Then each elected $\mathrm{CH}$ broadcasts an advertisement message to the rest of the nodes in the network to invite them to join their clusters. Based upon the strength of the advertisement signal, the non- 


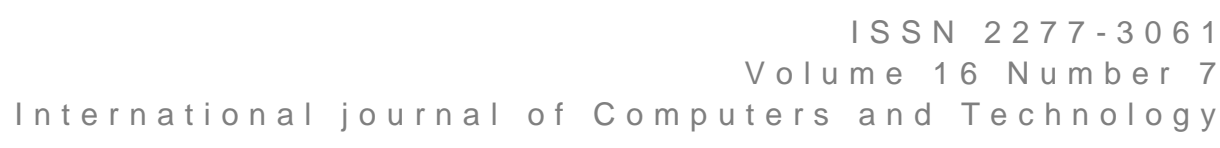

cluster head nodes decide to join the clusters. The non-cluster head nodes then informs their respective cluster- heads that they will be under their cluster by sending an acknowledgement message. After receiving the acknowledgement message, depending upon the number of nodes under their cluster and the type of information required by the system (in which the WSN is setup), the cluster-heads creates a TDMA schedule and assigns each node a time slot in which it can transmit the sensed data. The TDMA schedule is broadcasted to all the cluster-members. If the size of any cluster becomes too large, the cluster- head may choose another cluster- head for its cluster. The cluster-head chosen for the current round cannot again become the cluster-head until all the other nodes in the network haven't become the cluster-head.



Figure 2. Flowchart of the proposed methodology

2. Steady phase :- During the steady phase, the sensor nodes i.e. the non-cluster head nodes starts sensing data and sends it to their cluster-head according to the TDMA schedule. The cluster-head node, after receiving data from all the member nodes, aggregates it and then sends it to the mobile node in its trajectory. Mobile node will collect the data from the cluster heads of its sector and will forward them to the base station. After a certain time, which is determined a priori, the network again goes back into the setup phase and new cluster-heads are chosen. Each cluster communicates using different CDMA codes in order to reduce interference from nodes belonging to other clusters. The protocol takes into a number of assumptions which may create a lot of problems in the real-time systems. A few of these assumptions are as follows:

- The member nodes will communicate only with its $\mathrm{RH}$ or cluster head. They will never communicate with the mobile node or the base station.

- $\quad$ Each node has computational power to support different MAC protocols.

- Nodes always have data to send.

- $\quad$ Nodes located close to each other have correlated data.

- All nodes begin with the same amount of energy capacity in each election round, assuming that being a $\mathrm{CH}$ consumes approximately the same amount of energy for each node. 




- $\quad$ Different levels of power are used for different types of nodes. The member node will have less amplification energy in comparison with the cluster head and cluster head will further use less amplification energy in comparison with the mobile node.

\section{EXPERIMENTAL RESULTS}

The network area is of $80^{\star} 80 \mathrm{~m}$. The network area is divided into small regions of $40 * 40 \mathrm{~m}$ each. Each region has its own cluster head. The base station is fixed. There are 4 mobile nodes for each sector. Each sector will have 4 clusters inside it. Each mobile node will move along the boundary of the sector network. This section presents the simulation results of the work done and the proposed approach. The proposed approach has been implemented in MATLAB. The parameters required for the simulation is shown in the following Table 1.

Table 1. Simulation Parameters

\begin{tabular}{|c|c|}
\hline Network Parameters & Values \\
\hline Network Size & $80 \mathrm{~m}^{\star} 80 \mathrm{~m}$ \\
\hline Number of Sensor Nodes & 100 \\
\hline Initial Energy of Sensor Nodes & $0.5 \mathrm{~J}$ \\
\hline Packet Size & $6000 \mathrm{bits}$ \\
\hline $\begin{array}{c}\text { Data Aggregation Energy } \\
\text { consumption }\end{array}$ & $5 \mathrm{~nJ} / \mathrm{bit}$ \\
\hline $\begin{array}{c}\text { Amplification Energy } \\
\text { (Cluster to BS)dis }>=d 0\end{array}$ & Efs $=10 \mathrm{pJ} / \mathrm{bit} / \mathrm{m} 2$ \\
\hline $\begin{array}{c}\text { Amplification Energy } \\
\text { (Cluster to BS)dis }<\mathrm{do}\end{array}$ & Emp $=0.0013 \mathrm{pJ} / \mathrm{bit} / \mathrm{m} 2$ \\
\hline
\end{tabular}

Figure 3.explains the network model of size $80^{*} 80 \mathrm{~m}$ with 16 regions and 4 sectors. Each sector is having its own mobile node marked with pink color. The base station is available on the top of the network.

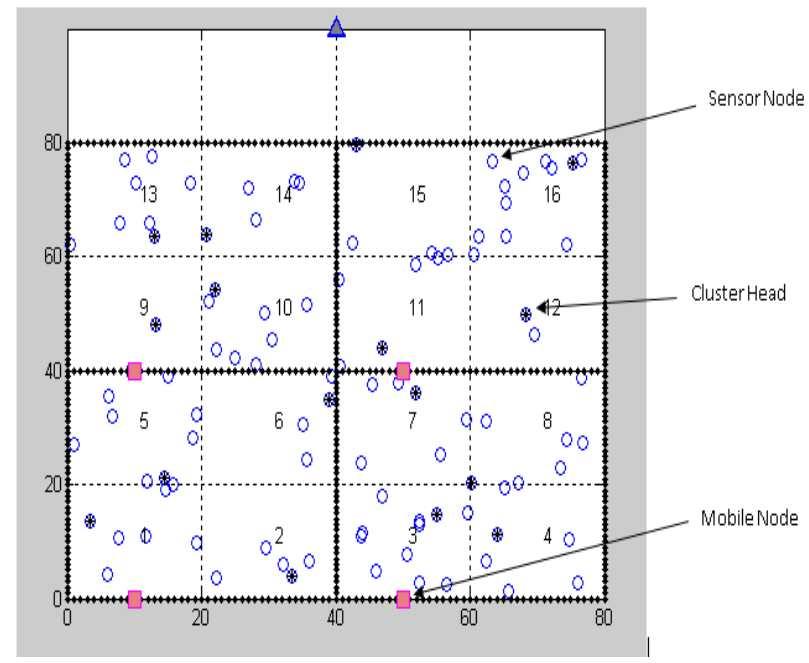

Figure 3. Network when nodes are Alive

We have used the homogenous network model for experimental purposes which states that all of the sensor nodes have got the same amount of energy. The mobile nodes are having unlimited amount of energy used in the following network, which include the network topology description and energy consumption model. Assume that $\mathrm{N}$ sensor nodes are randomly distributed in a square area $M \times M$. The feature of this scenario is as follows.

- All nodes are homogenous and energy constrained.

- All member nodes, cluster head and base station are immovable after deployment. Only mobile nodes can move in their sector to collect the data from cluster heads of corresponding cluster or region.

- Nodes are location unaware. But each node can compute the distance to other nodes based on the signal strength of received message.

- The nodes are having different level of transmission power for amplification of signals. 


\section{PERFORMANCE METRICS}

The performance metrics used in proposed work compared with the existing work are network life time, packets to cluster head and packets to base station, average energy consumption and average remaining energy.

\section{NETWORK LIFE TIME}

The lifetime of network depends upon the number of Dead \& Alive Nodes. The proposed scheme plays an important role to achieve better network life time. Table 2 illustrates the first node dead (FND), middle node dead (MND), last node dead (LND) of the existing work and the proposed work.

Table 2. Results taken at $1 \mathrm{~J}$ of energy

\begin{tabular}{|c|c|c|c|c|c|c|}
\hline \multirow{2}{*}{$\begin{array}{c}\begin{array}{c}\text { ENERGY = } \\
1 \mathrm{~J}\end{array} \\
\text { S. No. }\end{array}$} & \multicolumn{3}{|c|}{ EXISTING WORK } & \multicolumn{3}{|c|}{ CURRENT WORK } \\
\hline & FND & MND & LND & FND & MND & LND \\
\hline 1 & 2790 & 2957 & 3276 & 3074 & 5797 & 5892 \\
\hline 2 & 2772 & 2925 & 3990 & 4160 & 5663 & 5929 \\
\hline 3 & 2801 & 2950 & 3486 & 3138 & 5701 & 5874 \\
\hline 4 & 2802 & 2961 & 3397 & 3106 & 5651 & 6004 \\
\hline 5 & 2760 & 2932 & 3999 & 4745 & 5655 & 5856 \\
\hline 6 & 2795 & 2946 & 3252 & 3106 & 5651 & 6004 \\
\hline 7 & 2802 & 2934 & 3546 & 4745 & 5655 & 5856 \\
\hline 8 & 2757 & 2954 & 3686 & 3074 & 5797 & 5892 \\
\hline 9 & 2778 & 2935 & 3338 & 4160 & 5668 & 5929 \\
\hline 10 & 2773 & 2939 & 3907 & 3138 & 5701 & 5874 \\
\hline
\end{tabular}

Multiple number of experiments have been conducted at different levels of initial energy for evaluation and analysis of results. Initial energy has been set to 1 joule, 0.5 joule and 0.25 joule for different experiments. Table 3 illustrates the results of the existing work and the proposed work at 0.5 and 0.25 joule of initial energy. It is the clear from the results, that the network life time has been improved significantly.

Table 3. Results taken at $.50 \mathrm{~J}$ and $.25 \mathrm{~J}$ of energy

\begin{tabular}{|c|c|c|c|c|c|c|}
\hline ENERGY $=.50 \mathrm{~J}$ & \multicolumn{3}{|c|}{ EXISTING WORK } & \multicolumn{3}{|c|}{ CURRENT WORK } \\
\hline S. No. & FND & MND & LND & FND & MND & LND \\
\hline 1 & 1387 & 1467 & 1928 & 1538 & 2899 & 2947 \\
\hline 2 & 1391 & 1462 & 1859 & 2376 & 2873 & 2968 \\
\hline 3 & 1407 & 1466 & 1669 & 2391 & 2821 & 2918 \\
\hline 4 & 1389 & 1461 & 2030 & 1554 & 2826 & 3003 \\
\hline 5 & 1385 & 1474 & 1656 & 2373 & 2828 & 2929 \\
\hline 6 & 1380 & 1474 & 1985 & 1538 & 2899 & 2947 \\
\hline 7 & 1377 & 1453 & 2031 & 2081 & 2835 & 2966 \\
\hline 8 & 1380 & 1480 & 1757 & 1570 & 2851 & 2938 \\
\hline 9 & 1397 & 1470 & 1817 & 1518 & 2839 & 2930 \\
\hline 10 & 1399 & 1462 & 1773 & 1554 & 2826 & 3003 \\
\hline ENERGY $=.25 \mathrm{~J}$ & \multicolumn{3}{|c|}{ EXISTING WORK } & \multicolumn{3}{|c|}{ CURRENT WORK } \\
\hline S. No. & FND & MND & LND & FND & MND & LND \\
\hline 1 & 689 & 732 & 1028 & 1187 & 1415 & 1466 \\
\hline 2 & 677 & 731 & 906 & 778 & 1414 & 1502 \\
\hline
\end{tabular}




\begin{tabular}{|l|l|l|l|l|l|l|}
\hline 3 & 689 & 736 & 893 & 1187 & 1415 & 1466 \\
\hline 4 & 687 & 737 & 943 & 770 & 1450 & 1475 \\
\hline 5 & 690 & 737 & 840 & 1041 & 1418 & 1484 \\
\hline 6 & 696 & 737 & 866 & 786 & 1427 & 1470 \\
\hline 7 & 698 & 731 & 937 & 760 & 1420 & 1466 \\
\hline 8 & 680 & 732 & 894 & 1185 & 1424 & 1484 \\
\hline 9 & 694 & 740 & 884 & 1046 & 1440 & 1509 \\
\hline 10 & 692 & 732 & 1032 & 1044 & 1388 & 1503 \\
\hline
\end{tabular}

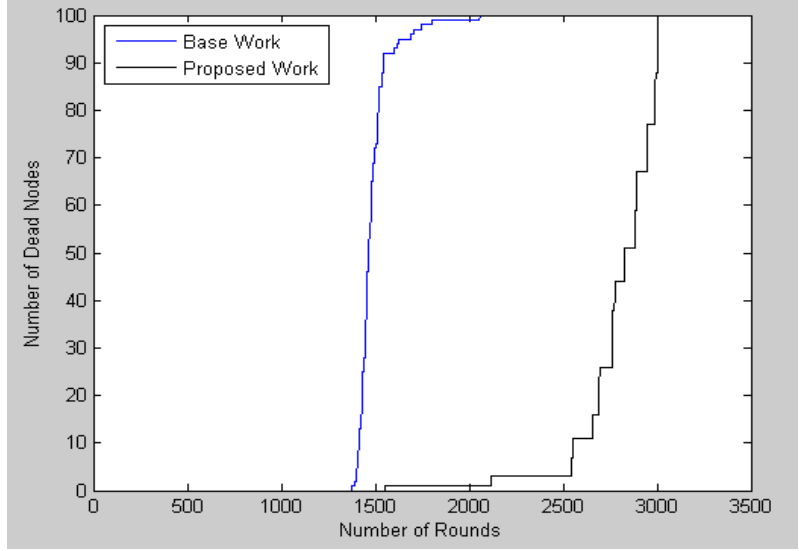

Figure 4 (a) Comparison of dead nodes

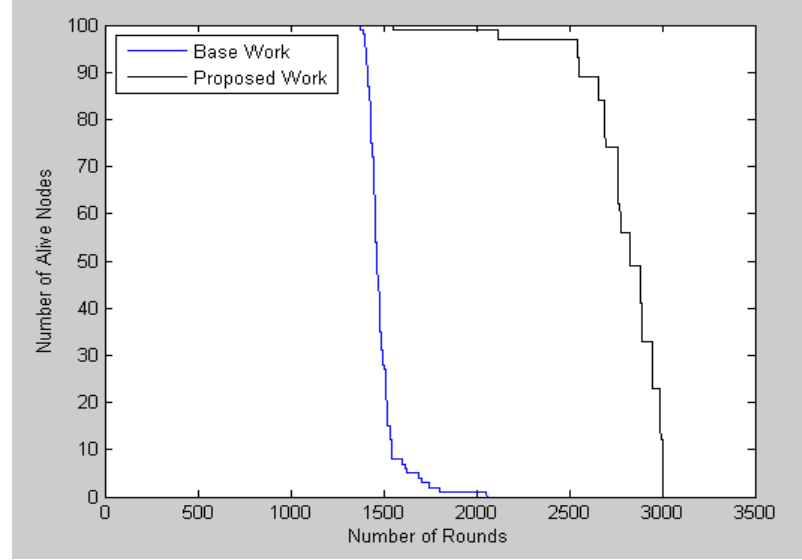

(b) Alive nodes comparison

The figure 4 (a) presents a comparison of the rounds achieved by all the simulated protocols when the all node dies. The nodes in the proposed work are alive for the longer period of time in comparison with the existing system which leads to the conclusion that the proposed mechanism is better than the existing approach. Figure 4 (b) shows the comparison of alive nodes of existing work with the proposed approach.

\section{THROUGHPUT}

The throughput is the fundamental parameter which checks the efficiency of the network in terms of data transfer. It is calculated as the successful number of message delivered over a communication channel and is measured in bits per second(bps).It represents the number of packets received by each node in the network over the time interval taken due packets delivery. Throughput is measured as:

$$
\text { Throughput }=\sum \frac{\text { Number of all delivered packets }}{\text { Receiving time interval length }}
$$

As illustrated in Figure 5 (a) the throughput comparison of existing and proposed algorithm. Throughput is also defined as the packets that successfully reached at the sink which means that the data received at sink is maximum with less packet dropping.



Figure 5 (a). Packets to base station

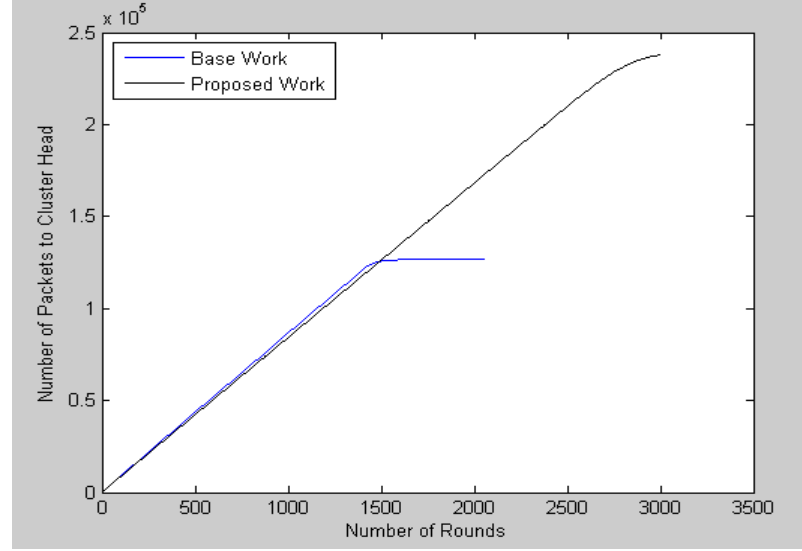

5 (b) Packets to cluster head 
The proposed algorithm achieves high throughput than existing algorithm as shown. Number of packets received by sink depends upon the number of alive nodes present in the network. More the Alive nodes, large number of packets are received by the destination sink node which improves the overall throughput of the network. Figure 5 (b) shows the packets sent to the cluster head.

\section{ENERGY CONSERVATION}

Figure 6 (a) represents the average energy consumed by the network during each round of the transmission time. The average energy consumed by the proposed algorithm is minimum as compared to the existing algorithm.



Figure 6 (a) Average energy consumed

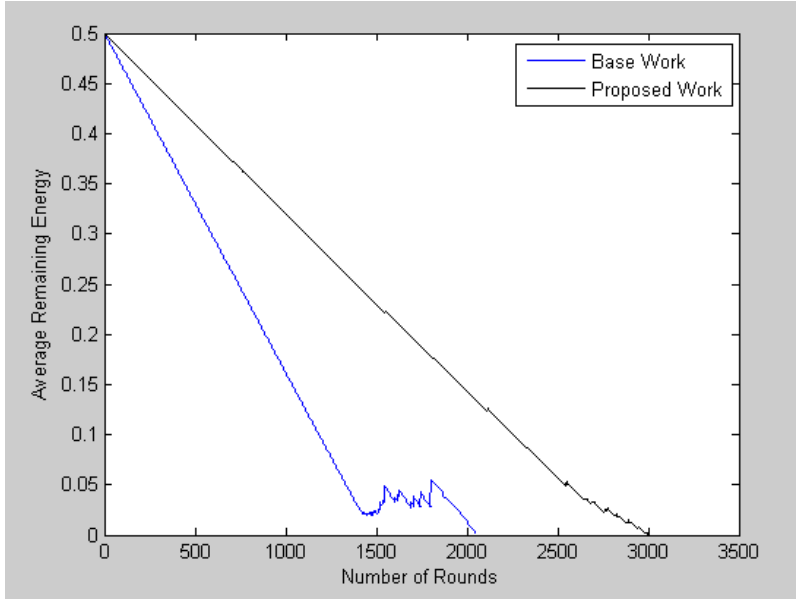

Figure 6 (b) Average remaining energy

We can see that almost all the sensors are alive for the proposed algorithm for quite long period of time, hence extending the network efficiency. For existing algorithms, as no. of dead sensors will increase, their efficiency will decrease. The energy consumption in the proposed approach is slower in comparison with the existing approach. It leads to the more number of rounds which means more lifetime. The consumption of the energy has been reduced which has improved the network lifetime. In this subsection the figure 6 (b) presents a comparison of the average remaining energy of the existing work and the proposed work. As we can see from the figure $6(\mathrm{~b})$, it is clear that the average remaining energy of all the sensor nodes in the proposed approach is greater than 0 for the maximum number of rounds. The sensor nodes in the proposed approach are active for the longer duration and will keep on sending the data to the cluster heads and finally to the base station.

\section{CONCLUSION}

This paper included the region based clustering, cluster head selection and energy efficient communication using static base station and movable mobile nodes. Since it was earlier proposed that clustering improves the network lifetime. We modified the region based clustering by dividing the network area into $\mathrm{n}$ regions with cluster head chosen for each region and proposed a new method for cluster head selection having less computational complexity. It was also found that the modified approach has improved performance to that of the other clustering approaches. We have used the mobile nodes for each section with controlled trajectory path as a reference to compare the performance of each of the clustering methods. It was found that the proposed algorithm gives a much-improved network lifetime as compared to existing work. In this thesis, the nodes are divided into multiple regions depending upon the network area. Multiple transmission power levels have been used for energy saving architecture. This algorithm is applied prior to a cluster algorithm to improve the performance of the clustering algorithm without affection the performance of individual sensor nodes. As a result, the network lifetime has been prolonged. By analyzing the results and graphs, we have reached up to the solution that by changing the clustering and communication mechanism, the overall lifetime of the complete network can be improved. In all of the methods discussed above the energy parameter is taken into consideration only during cluster head selection. The performance may be increased by considering energy as a parameter during clustering itself. Multiple number of experiments have been conducted to evaluate the effectiveness of the experiments. Due to the potential deployment in uncontrolled and harsh environments and due to the complex architecture, wireless sensor networks are and will be prone to a variety of malfunctioning In future work, we intend to calculate the detection accuracy for the nodes in the Wireless Sensor Network where detection accuracy depicts the ratio of the number of faulty sensors detected to the total number of faulty sensors in the network. In the present work, we have improved the overall lifetime of the network but we will also analyze the faults occur inside the WSN in the future in real time scenarios.

\section{REFERENCES}

[1] Y. Xiuwu, Fan Feisheng Zhou Lixing and Z. Feng, "WSN Monitoring Area Partition Clustering Routing Algorithm for Energy-Balanced," IEEE, pp. 80-84, 2016.

[2] Dan Liu, Qian Zhou, Zhi Zhang and Baoling Liu, "Cluster-Based Energy-Efficient Transmission Using a New Hybrid Compressed Sensing in WSN," IEEE, 2016. 




[3] Li Li , Zhu Yafei, Duan Xiaoyi and Shi Guozhen, "Research on Secure Communication Scheme of Clustered WSN Based on Dynamic Key," IEEE, pp. 833-837, 2016.

[4] S. Bera, S. Misra, Sanku Kumar Roy and Mohammad S. Obaidat, "Soft-WSN: Software-Defined WSN Management System for loT Applications," IEEE, pp. 1-8, 2016.

[5] N. A. M. Alduais, J. Abdullah, J. Abdullah, A. Jamil and L. Audah, "An Efficient Data Collection and Dissemination for IOT based WSN," IEEE, 2016.

[6] O. Singh, V. Rishiwal and M. Yadav, "Energy Trends of Routing Protocols for H-WSN," IEEE, 2016.

[7] R. Kumari and . P. Nand, "Performance Comparison of various Routing Protocols in WSN and WBAN," IEEE, pp. 427-431, 2016.

[8] Hector Kaschel and ohanna Ortega , "Energy efficiency in routing protocols applied to WSN," IEEE, 2016.

[9] Asdianur Hadi and Ida Wahidah, "Delay Estimation using Compressive Sensing on WSN IEEE 802.15.4," IEEE, pp. 192-197, 2016.

[10] Mohd Zaki Shahabuddin, Halabi Hasbullah and Izzatdin A Aziz, "eliminary Framework of Topology Control Algorithm Ahieve Node's Energy Efficiency," IEEE, pp. 259-263, 2016.

[11] M. Shaiful Azrin , Md Alimon, L.M. Kamarudin and Goh Chew Cheik, "Connecting WSN to Google Fusion Tables, a REST Database for Data Storage," IEEE, pp. 342-347, 2016.

[12] Abhaykumar L. Gupta and Narendra Shekokar , "A Novel Approach to Improve Network Lifetime in WSN by Energy Efficient Packet Optimization," IEEE, 2016.

[13] B. Bengherbia, S. Chadli, M. Ould Zmirli and A. Toubal, "A MicroBlaze Based WSN Sink Node Using XBee Transceiver," IEEE, pp. 831-834, 2016.

[14] Gagandeep Kaur, Deepali and Rekha Kalra, "Improvement and Analys Security of WSN From Passive Attack," IEEE, pp. 4520-425, 2016.

[15] M. Wu, H. Liu and Q. Min, "Lifetime Enhancement by Cluster Head Evolutionary Energy Efficient Routing Model for WSN," IEEE, pp. 545-548, 2016.

[16] Roman Lara-Cueva, Rodolfo Gordillo, Liliana Valencia and Diego S. Ben, "Determining the Main CSMA Parameters for Adequate Performance of WSN for Real-time Volcano Monitoring System Applications," IEEE, pp. 1-9, 2016.

[17] Sanaa. S. Abd El dayem and M. R. M. Rizk, "An Efficient Authentication Protocol and Key Establishment in Dynamic WSN," IEEE, pp. 178-182, 2016.

[18] Carlos Valdivieso, Francisco Novillo, Jorge Gomez and Daniel Dik, "Centralized Channel Assignment Algorithm for WSN Based on Simulated Annealing in Dense Urban Scenarios," IEEE, 2016.

[19] Jai Prakash Prasad, and Suresh Chandra Mohan , "Global Wireless Tele-Communication WSN's Architecture Using N-Tier Spherical GRID Routing," IEEE, pp. 108-112, 2016.

[20] Swathi N, Santosh Kumar S, R. Sunil Kumar KN, and Rajendra Prasad P , "Zone Based Hierarchical Energy Efficient Clustering Scheme for WSN," IEEE, pp. 136-140, 2016.

[21] Praveen Kumar K.V, , M.K Banga,, Udaya Rani V, B.M Thippeswamy, and K.R Venugopal , "EBDRA: Energy Balanced Dynamic cluster Routing Approach for WSN," IEEE, pp. 141-145, 2016.

[22] Pavithra.H, , Shivashankar and Poornima G.R, "An Efficient Mobile Sink path Selection approach for WSN's," IEEE, pp. 151-155, 2016.

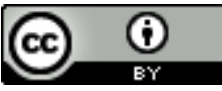

This work is licensed under a Creative Commons Attribution 4.0 International License.

\section{DOI: $10.24297 /$ ijct.v16i7.6454}

